Check for updates

Cite this: RSC Adv., 2018, 8, 39203

Accepted 19th November 2018

DOI: $10.1039 / \mathrm{c} 8 \mathrm{ra0} 8509 a$

rsc.li/rsc-advances

\section{Facile synthesis of $\mathrm{AgBiS}_{2}$ nanocrystals for high responsivity infrared detectors $\uparrow$}

\author{
Chun Hin Mak, Jiasheng Qian, Lukas Rogée, Wai Kin Lai and Shu Ping Lau (D) * \\ $\mathrm{AgBiS}_{2}$ nanocrystals are emerging optoelectronic materials due to their solution-processability, earth \\ abundance and non-toxic properties. We report a facile method to prepare $\mathrm{AgBiS}_{2}$ nanocrystals in \\ ambient conditions. The nanocrystals are of high crystallinity and without byproducts, which make them \\ suitable for solution processable optoelectronic devices. They were incorporated into graphene \\ transistors for their near infrared detection application. Photodetectors with a high photo-responsivity of \\ $10^{5} \mathrm{~A} \mathrm{~W}^{-1}$ for $895 \mathrm{~nm}$ wavelength at a low operation voltage of $0.1 \mathrm{~V}$ were demonstrated.
}

\section{Introduction}

Over the past decade, lead chalcogenide nanocrystals (NCs) such as lead sulphide (PbS) have been intensively studied for solar cells and infrared (IR) detection. ${ }^{1-6}$ Due to their high absorption coefficients and solution dispersibilities, high performance solar cells and hybrid phototransistors have been demonstrated. However, the toxicity of lead causes long term environmental and biological issues, ${ }^{7}$ which limits its applications. Recently, $\mathrm{AgBiS}_{2} \mathrm{NCs}$, as a non-toxic and high stability material, have been utilized as the active material for solar cells showing a power conversion efficiency of $6.3 \% .^{8}$ The work was recognized as the first demonstration of efficient inorganic nanocrystal-based solar cells which simultaneously fulfilled the advantages of non-toxicity, abundance, low-temperature and solution processability. However, even though the $\mathrm{AgBiS}_{2} \mathrm{NCs}$ exhibited a high infrared external quantum efficiency in the aforementioned solar cells with a bandgap fall in the infrared region (1.0-1.25 eV), ${ }^{8,9}$ the investigation in near infrared (NIR) detection is scarce.

Graphene transistors have been used for IR detection but the responsivity is very low $\left(<10 \mathrm{~mA} \mathrm{~W}^{-1}\right) .{ }^{10-12}$ This is due to the low optical absorption of graphene, ${ }^{\mathbf{1 3 , 1 4}}$ which makes graphene transistor not favourable for photodetection. Graphene itself, however, provides a high mobility for charge carriers. ${ }^{15}$ Therefore graphene transistor has been hybridized with various semiconducting NCs such as $\mathrm{PbS}$ and halide perovskite $\left(\mathrm{CsPbX}_{3}\right)$ for broad range photodetection. ${ }^{16,17}$ The photoresponsivities of $\mathrm{PbS}$ and $\mathrm{CsPbX}_{3}$ based graphene transistors could reach $10^{7}$ and $10^{8} \mathrm{~A} \mathrm{~W}^{-1}$ respectively. Recently another non-toxic Si NCs was hybridized with graphene and showed

Department of Applied Physics, The Hong Kong Polytechnic University, Hung Hom, Kowloon, Hong Kong SAR. E-mail: apsplau@polyu.edu.hk

$\dagger$ Electronic supplementary information (ESI) available. See DOI: 10.1039/c8ra08509a a photo-responsivity of $10^{8}$ to $10^{9} \mathrm{~A} \mathrm{~W}^{-1}$ under $375 \mathrm{~nm}$ to $1870 \mathrm{~nm}$ light illumination. The devices also exhibited midinfrared photo-responsivity of $0.22-44.9 \mathrm{~A} \mathrm{~W}^{-1}$ in 2.5 to 3.9 $\mu \mathrm{m} .{ }^{18}$ Both works evidence that graphene transistor is an ideal platform to amplify the intrinsic optoelectronic properties of the NCs.

It is also favorable that the synthesis of functional NCs becomes simple and cost effective. Herein we present a facile synthetic route for $\mathrm{AgBiS}_{2}$ NCs with a much shorter processing time under ambient conditions as compared to the conventional hot injection method. ${ }^{8}$ The $\mathrm{AgBiS}_{2}$ NC solution was spincoated onto a graphene transistor for $895 \mathrm{~nm}$ light detection. A high photo-responsivity of $1.24 \times 10^{5} \mathrm{~A} \mathrm{~W}^{-1}$ was achieved by a small operation voltage of $V_{\mathrm{DS}}=0.1 \mathrm{~V}$. Our findings demonstrate that the $\mathrm{AgBiS}_{2}$ NCs can be synthesized by a facile technique for optoelectronic application.

\section{Experimental}

\section{Chemicals and reagents}

Oleic acid (OA), octanoic acid (OctA), octylamine (OTA), dodecylamine (DDA), tetrabutylammonium iodide (TBAI) and sulfur powder were purchased from Sigma Aldrich. Silver nitride $\left(\mathrm{AgNO}_{3}\right)$ and bismuth(III) nitrate pentahydrate $\left(\mathrm{Bi}\left(\mathrm{NO}_{3}\right)_{3} \cdot 5 \mathrm{H}_{2} \mathrm{O}\right)$ were purchased from Aladdin. Toluene, methanol and ethanol were ACS reagent grade. All chemicals were used without any purification.

\section{Materials synthesis}

Typically, $0.1 \mathrm{mmol} \mathrm{AgNO}_{3}$ and $0.1 \mathrm{mmol} \mathrm{Bi}\left(\mathrm{NO}_{3}\right)_{3} \cdot 5 \mathrm{H}_{2} \mathrm{O}$ were heated $\left(120{ }^{\circ} \mathrm{C}\right)$ and stirred by a heating mantle in a flask containing $10 \mathrm{~mL}$ oleic OA (or OctA) until fully dissolved and the color turned pale yellow. Then $0.1 \mathrm{mmol}$ sulfur powder was dissolved and stirred into a long chain length amine such as OTA (or DDA). After the dissolution of all the precursors, $1 \mathrm{~mL}$ $\mathrm{Ag} / \mathrm{Bi}$ and $1 \mathrm{~mL}$ sulfur were mixed under vigorous stirring in 
room temperature and atmosphere. The color of the whole mixture turned black immediately and the $\mathrm{AgBiS}_{2}$ nanocrystals were formed. After the synthesis process, the $\mathrm{AgBiS}_{2}$ nanocrystals were washed by precipitation/dispersion in a toluene/ ethanol mixture via centrifugation. The $\mathrm{AgBiS}_{2} \mathrm{NCs}$ were dispersed in toluene after washing three times.

Graphene was prepared by using a 2-inch copper foil (Alfa Aesar, $0.025 \mathrm{~mm}$ thick) in a plasma enhanced chemical vapor deposition chamber (Aixtron CVD). The synthesis process of graphene included the following steps: the $\mathrm{Cu}$ foil was annealed in a mixture gas (Ar: $250 \mathrm{sccm}, \mathrm{Ar} / \mathrm{H}_{2}: 1000 \mathrm{sccm}$ ) at constant pressure of 25 mbar. The annealing process was carried out at $1020{ }^{\circ} \mathrm{C}$ for 15 minutes. Then methane gas $(15 \mathrm{sccm})$ was injected into the chamber. The graphene formation was lasted for 5 minutes. Finally, the sample was cooled down to $150{ }^{\circ} \mathrm{C}$ under Ar flow.

\section{Device fabrication and electrical measurement}

First of all, $\mathrm{Cr} / \mathrm{Au}$ metal electrodes were patterned on $\mathrm{Si} / \mathrm{SiO}_{2}$ substrate $\left(\mathrm{SiO}_{2}, 300 \mathrm{~nm}\right.$ thick) by photolithography and then DC magnetron sputtering deposition. A thin layer of poly(methyl methacrylate) (PMMA) was spun-coated on top of graphene/ copper foil and heated at $150{ }^{\circ} \mathrm{C}$ for 30 minutes. Then the PMMA/graphene/copper composite was let to float on ammonium persulfate/deionized water solution with concentration 2 $\mathrm{g} / 60 \mathrm{~mL}$. After the copper foil was completely etched, the PMMA/graphene composite was cleaned with DI water for two times and transferred onto the patterned $\mathrm{Cr} / \mathrm{Au}$ electrodes and dried at $120{ }^{\circ} \mathrm{C}$ for 30 minutes. Finally, the samples were immersed into acetone for 6 hours to remove PMMA and the graphene transistor was ready for use.

For $\mathrm{AgBiS}_{2}$ /graphene hybrid, $\mathrm{AgBiS}{ }_{2}$ NCs were dropped onto the graphene transistor and spin-coated with $2000 \mathrm{rpm}$ for $30 \mathrm{~s}$. Then, $5 \mathrm{mg} \mathrm{mL}{ }^{-1} \mathrm{TBAI} / \mathrm{methanol}$ solution was drop onto the $\mathrm{AgBiS}_{2}$ NCs to replace the long organic ligand to single atom ligand iodine, then spin-coated with $2000 \mathrm{rpm}$ for $30 \mathrm{~s}$. The device was rinsed with methanol followed by toluene. The above procedure is for a cycle of layer-by-layer (LBL) $\mathrm{AgBiS}_{2}$ deposition. The $\mathrm{AgBiS}_{2}$ NCs were deposited by 5 (5-layer) and 10 (10-layer) cycles of LBL deposition respectively. The thickness of the $\mathrm{AgBiS}_{2}$ film for 10 cycles deposition is about $72 \mathrm{~nm}$. The transistors were annealed at $150{ }^{\circ} \mathrm{C}$ for 90 minutes in a $\mathrm{N}_{2}$-filled glove box to remove the surface adsorbates.

All the electrical measurements for both the control graphene transistor and the $\mathrm{AgBiS}_{2}$ /graphene transistor were carried out with a Keithley 4200-SCS at room temperature in a nitrogen filled glove box with $\mathrm{O}_{2}, \mathrm{H}_{2} \mathrm{O}<10 \mathrm{ppm}$. For infrared detection, a light emitting diode with emission wavelength of $895 \mathrm{~nm}$ was used to illuminate the transistor channel.

\section{Materials characterization}

Transmission electron microscopy (TEM), selected area electron diffraction (SAED) and energy-dispersive X-ray spectroscopy (EDX) measurements were performed by JEOL Model JEM2100F Field Emission Electron Microscope STEM. Carbon coated copper grids were used as X-ray diffraction (XRD) measurement was conducted by the Rigaku SmartLab X-ray Diffractometer. Cross-sectional image of the $\mathrm{AgBiS}_{2}$ thickness was observed by Tescan VEGA3 Scanning Electron Microscope. Raman spectrum of graphene was measured by LabRAM HR 800 Raman Spectrometer. Fourier Transform Infrared (FTIR) spectroscopy of the NCs on quartz was obtained by The Bruker Vertex-70 FTIR.

\section{Results and discussion}

In our experiment, $0.1 \mathrm{mM} \mathrm{Ag} / \mathrm{Bi}$ oleate solution and $0.2 \mathrm{mM}$ sulfur amine solution were mixed under rigorous stirring, resulting to a black solution. Fig. 1A shows the photograph of the three solutions and revealing their colours. Besides the OA and DDA ligand pair used, two other pairs namely OA and OTA, OctA and OTA, were also employed to fabricate the NCs with similar result. Detailed synthesis information is described in the materials synthesis section.

TEM was used to characterize the size distribution and crystal structure of the NCs produced by the OA/DDA ligand. The size distribution of the NCs is ranging from 7 to $15 \mathrm{~nm}$ with the most frequent size at around $8 \mathrm{~nm}$ as shown in Fig. 1B. TEM image and SAED pattern in Fig. 1C depict that the NCs are having a cubic structure. SAED pattern shows clear rings of the (111), (200), and (220) crystal planes. The results are in good agreement with the XRD pattern of the sample, revealing three major peaks corresponding to the plane (111), (200), and (220) of $\mathrm{AgBiS}_{2}$ as shown in Fig. S1. $\dagger$ Fig. 1D shows the high resolution TEM image of a single NC revealing the interplanar distance of $0.32 \mathrm{~nm}$ for the atomic plane of (111). The composition of the product was analysed by EDX. The result reveals that the atomic ratio of $\mathrm{Ag} / \mathrm{Bi} / \mathrm{S}$ is very close to $1: 1: 2$ and no oxygen is detected. Similar characterization results were obtained when the ligand pairs changed to OA/OTA and OctA/OTA

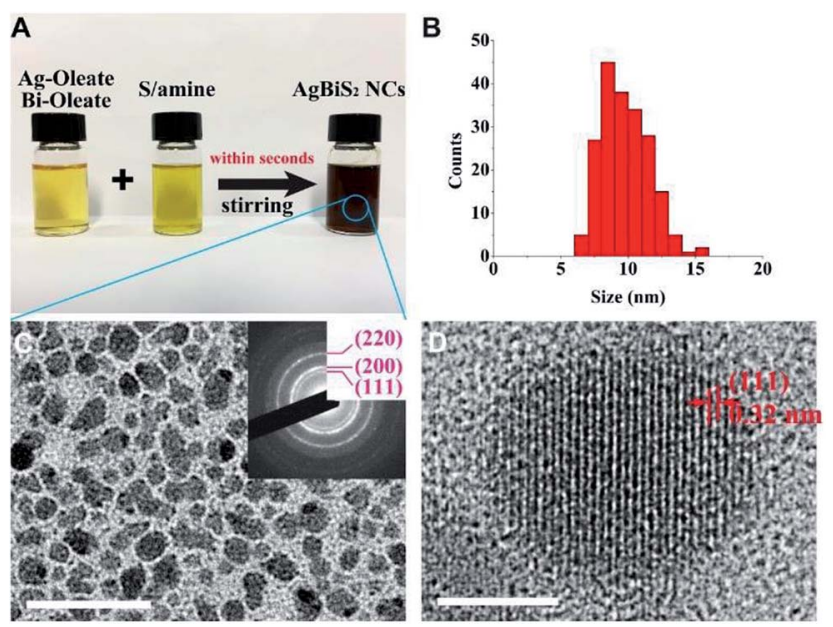

Fig. 1 Characterizations of the $\mathrm{AgBiS}_{2} \mathrm{NCs}$ synthesized by OA/DDA. (A) Photograph of the $\mathrm{Ag} / \mathrm{Bi}$-oleate, sulfur in amine and the $\mathrm{AgBiS}_{2}$ NCs. (B) Size distribution profile of the $\mathrm{AgBiS}_{2} \mathrm{NCs}$. (C) TEM image of the $\mathrm{AgBiS}_{2} \mathrm{NCs}$. Scale bar is $50 \mathrm{~nm}$. Inset: SAED pattern. (D) High resolution TEM image of a single $\mathrm{AgBiS}_{2} \mathrm{NC}$. Scale bar is $5 \mathrm{~nm}$. 

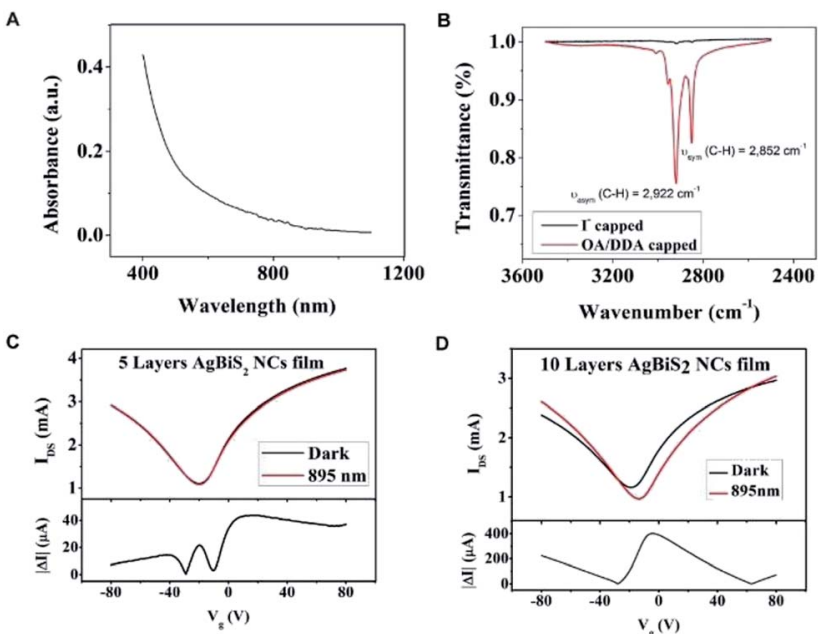

$D$

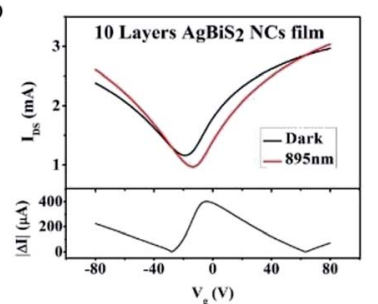

Fig. 2 (A) UV-vis absorption spectrum of $\mathrm{AgBiS}_{2} \mathrm{NCs}$ in toluene. (B) The FTIR spectra of the films before (OA/DDA capped) and after (Icapped) solid state ligand exchange. (C and D) The transfer characteristic curves with/without $895 \mathrm{~nm}$ light illumination (UP) and photocurrent change (down) for the hybrid transistor with 5 layers NC film (C) and 10 layers NC film (D), illumination intensity $=10.43 \mathrm{~mW}$ $\mathrm{cm}^{-2}$.
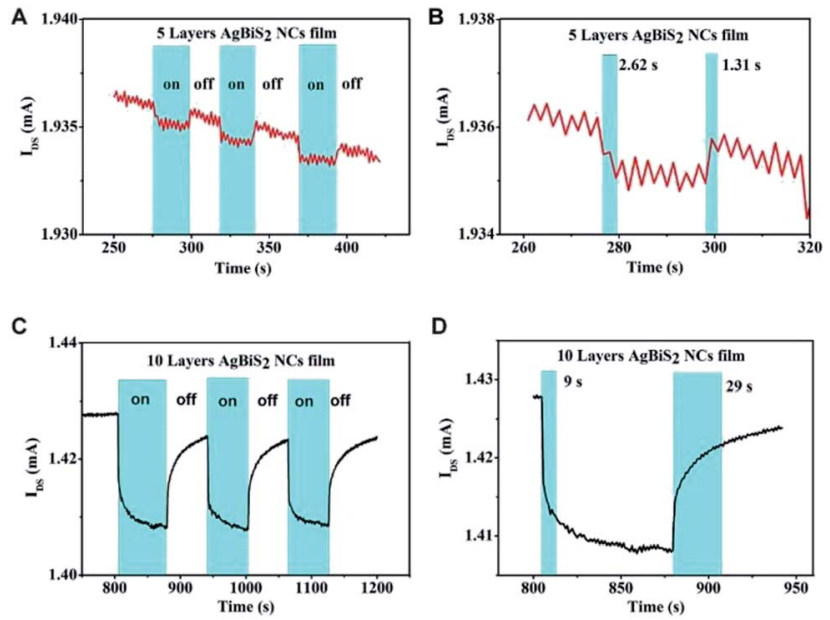

Fig. 3 (A) The photocurrent response of the $5 \mathrm{~L}$ device. (B) Reaction time and recovery time of the $5 \mathrm{~L}$ device. (C) The photocurrent response of the $10 \mathrm{~L}$ device. (D) Reaction time and recover time of the $10 \mathrm{~L}$ device. The light intensity of $12.28 \mu \mathrm{W} \mathrm{cm}{ }^{-2}$ was used for all the measurements.

(Fig. S2 and $\mathrm{S} 3 \dagger$ ). The result indicates that the choice of the ligand - pair is independent on the synthesis process.

Cubic $\mathrm{AgBiS}_{2}$ was reported to have the bandgap of about $0.8 \mathrm{eV}$ in the bulk form and about $1.0-1.25 \mathrm{eV}$ in the nanocrystalline form. ${ }^{8,9}$ Fig. 2 A shows the absorption spectrum of the NCs in toluene, revealing a broad absorption covering $400 \mathrm{~nm}$ to $1000 \mathrm{~nm}$. The graphene was prepared by chemical vapor deposition and characterized by Raman scattering. The graphene is monolayer and no defect peaks was found (Fig. S4 $\dagger$ ). The intrinsic transfer characteristic curve from $-80 \mathrm{~V}$ to $80 \mathrm{~V}$

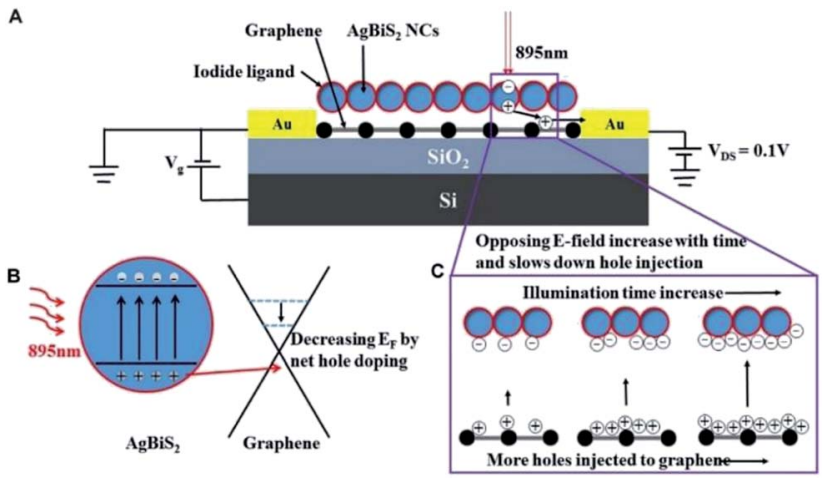

Fig. 4 (A) The schematic illustration of the $\mathrm{AgBiS}_{2} \mathrm{NCs} /$ graphene photodetector. The wavelength of light is $895 \mathrm{~nm}$. (B) The schematic diagram for charge generation at the $\mathrm{AgBiS}_{2} \mathrm{NCs} /$ graphene heterojunction under illumination. (C) The schematic diagram for opposing field at the $\mathrm{AgBiS}_{2} \mathrm{NCs} /$ graphene heterojunction, thicker film usually lead to a longer response time and stabilization time.

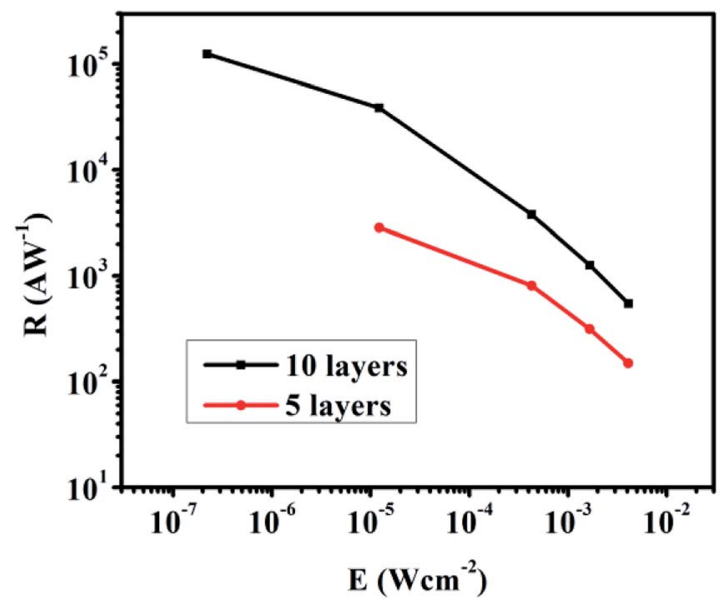

Fig. 5 Photo-responsivities of the $5 \mathrm{~L}$ and $10 \mathrm{~L}$ devices under $895 \mathrm{~nm}$ illumination.

was characterized before the deposition of the NCs (Fig. S5 $\dagger$ ). The Dirac point is located at $5 \mathrm{~V}$ which indicates a slight p-type characteristic. It is noteworthy that the long aliphatic chain molecule ligands such as OA/DDA are insulating which inhibits the charge transfer through the NCs to the surrounding. ${ }^{19,20}$ Therefore, the surface ligand exchange was changed to iodide ligand to enhance the surface's conductivity. Fourier-transform infrared spectroscopy (FTIR) was measured on the samples before and after the ligand exchange. Fig. 2B shows the trans-

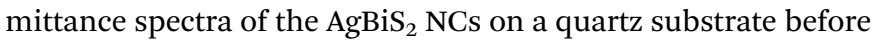
and after the ligand exchange. The $\mathrm{C}-\mathrm{H}$ vibrations $\left(2922 \mathrm{~cm}^{-1}\right.$ and $2852 \mathrm{~cm}^{-1}$ ) are nearly disappeared after the ligand exchange, which implies the removal of the long aliphatic chain ligand OA/DDA from the NCs surface. ${ }^{2,21}$

The layer-by-layer deposition was employed to deposit the NC film and exchange the ligands to provide different thicknesses of the $\mathrm{AgBiS}_{2}$ films on the transistors. ${ }^{8}$ We prepared two graphene transistors with 5-layer (5L) and 10-layer (10L) NC 
Table 1 Comparison of the $\mathrm{AgBiS}_{2}$ photodetector with $\mathrm{PbS}$ and $\mathrm{Si}$ NCs based hybrid phototransistors ${ }^{a}$

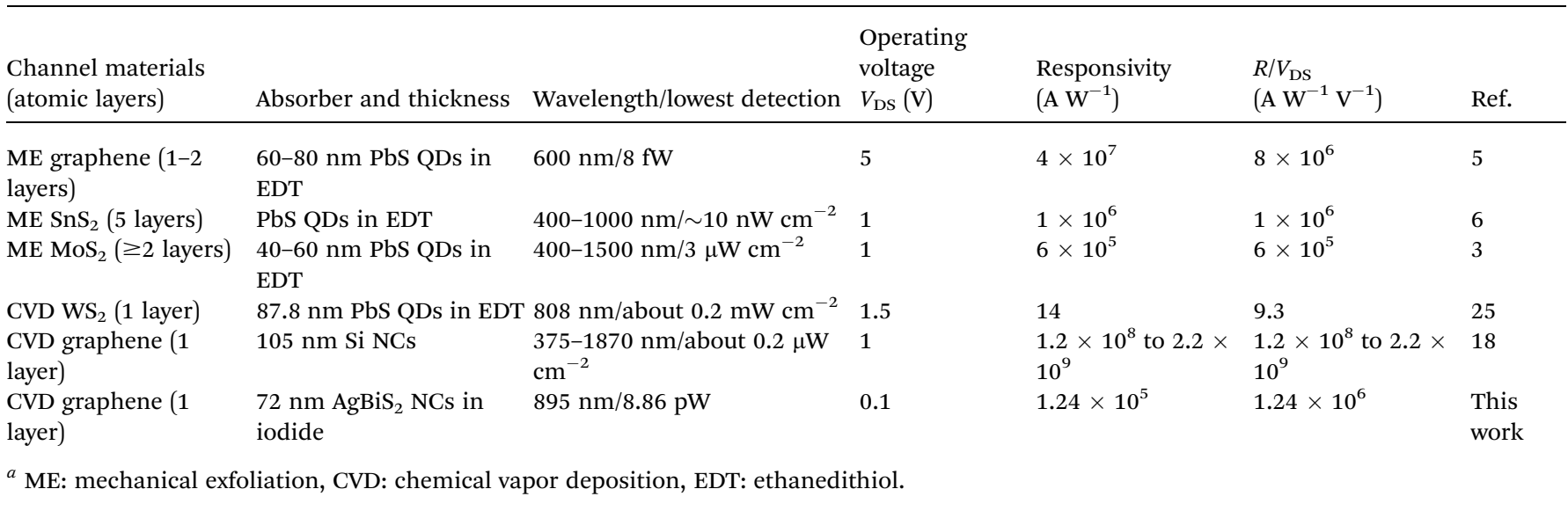

films. According to the SEM measurement, the film thickness of the 10L sample is about $72 \mathrm{~nm}$ (Fig. S6 $\dagger$ ). Fig. 2C and D show the transfer characteristic curves of the $5 \mathrm{~L}$ and $10 \mathrm{~L}$ devices with and without $895 \mathrm{~nm}$ light illumination. An applied voltage of $0.1 \mathrm{~V}$ (i.e. $V_{\mathrm{DS}}=0.1 \mathrm{~V}$ ) was applied to the drain and source electrodes. The Dirac point of the $5 \mathrm{~L}$ and $10 \mathrm{~L}$ devices are in the negative voltage region and indicating their n-type characteristic. Under light illumination, the Dirac point of $10 \mathrm{~L}$ device is obviously shifted to the positive voltage side which indicates a net hole doping to the graphene channel. As the quantity of the main charge carrier electron is reduced, the decrease in current are resulted. As an evidence, the currents under illumination in Fig. 2C and D at $V_{\mathrm{g}}=0 \mathrm{~V}$ are also decreased as compared to the dark current. The absolute photocurrent of these devices was calculated by subtracting of the current of the illuminated and unilluminated devices. The high absolute photocurrents values around $V_{\mathrm{g}}=0 \mathrm{~V}$ were observed in both devices. Therefore the photo-responsivity measurement was characterized at $V_{\mathrm{g}}=0 \mathrm{~V}$ and $V_{\mathrm{DS}}=0.1 \mathrm{~V}$.

Fig. 3 shows the temporal photo-response of the $5 \mathrm{~L}$ and $10 \mathrm{~L}$ devices under an intensity of $12.28 \mu \mathrm{W} \mathrm{cm}^{-2}$. The response time and current change are highly repeatable for both devices. The current change is about $1 \mu \mathrm{A}$ for the $5 \mathrm{~L}$ device and about $20 \mu \mathrm{A}$ for the 10L device (Fig. 3A and $\mathrm{C}$, respectively). The response time of the $5 \mathrm{~L}$ device is $2.62 \mathrm{~s}$, which is nearly twice of the recovery time (Fig. 3B). In addition, the response time becomes longer when increasing the NC film thickness. The response time of the $5 \mathrm{~L}$ device is $9 \mathrm{~s}$, while the recovery time is $29 \mathrm{~s}$ (Fig. 3D). The results clearly demonstrate that under the same light intensity illumination, the thicker absorber film will lead to higher photocurrent change but longer stabilization time for response and recovery, vice versa.

To illustrate the difference of stabilization time, the schematic diagram of charge transfer mechanism is shown in Fig. 4. When the light illuminates the graphene transistors, electronhole pairs (EHPs) are generated and holes are transferred to the graphene channel (Fig. 4B). This results in the decrease of Fermi level in the graphene, leading to positive shift of the Dirac point. According to the photo-gating effect, the holes will be accumulated in the graphene channel while electrons are trapped into the $\mathrm{AgBiS}_{2} \mathrm{NC}$ film. Since the transistors were annealed and characterized in $\mathrm{N}_{2}$-filled glovebox, the surface adsorbates such as moisture and oxygen were removed as much as possible. So the trapping of charge carriers are likely come from the surface defects of the NCs. ${ }^{5,18,22}$ Under light illumination, the current is changing with the increasing holes concentration in the graphene until equilibrium is reached. ${ }^{22,23}$ However, the holes at the graphene channel generate an opposing electric field to the absorber and hinder the rate of holes injection. As a result, the response time will be increased. Considering the thickness dependent light absorption of the absorber, fewer holes are generated from the NC film under illumination for the $5 \mathrm{~L}$ device due to weaker absorption. Fewer holes will be injected to the graphene and weaken the opposing field as compared with the 10L device. When the light is off, the trapped electrons are released and recombined with the holes. The 10L device's have thicker NC film and hence it trapped more electrons than the $5 \mathrm{~L}$ device. The total time of releasing the electrons is longer in the $10 \mathrm{~L}$ device as compared to the $5 \mathrm{~L}$ one. Consequently the temporal photo-response and recovery time of the $5 \mathrm{~L}$ device is faster than the $10 \mathrm{~L}$ device, but the photocurrent change is smaller than the $10 \mathrm{~L}$ device. ${ }^{24}$

The photo-responsivity $R$ is defined as the output photocurrent $I_{\mathrm{ph}}$ divided by the incident light power $P_{\mathrm{in}}{ }^{22,23}$ i.e.

$$
R=\frac{I_{\mathrm{ph}}}{P_{\mathrm{in}}}=\frac{\Delta I_{\mathrm{DS}}}{W L E_{\mathrm{in}}}=\frac{C_{\mathrm{i}} \mu \Delta V_{\mathrm{G}} V_{\mathrm{DS}}}{L^{2} E_{\mathrm{in}}}
$$

where $I_{\mathrm{ph}}$ is the photocurrent, $P_{\text {in }}$ is the incident power, $W$ and $L$ are the channel width $(800 \mu \mathrm{m})$ and channel length $(5 \mu \mathrm{m})$ respectively. $E_{\mathrm{in}}$ is the light intensity, $C_{\mathrm{i}}$ is the capacitance of the gate dielectric per unit area, $V_{\mathrm{DS}}$ and $I_{\mathrm{DS}}$ are the drain source voltage and current respectively, $\mu$ is the field effect mobility and $\Delta V_{\mathrm{G}}$ is the gate voltage shift. $h$ is Planck's constant, $c$ is the speed of light, $\lambda$ is the wavelength of the incident photons, and $q$ is the elementary charge. Fig. 5 shows the photo-responsivity of the $5 \mathrm{~L}$ and $10 \mathrm{~L}$ devices as a function of light intensity. A maximum responsivity of $2.85 \times 10^{3} \mathrm{~A} \mathrm{~W}^{-1}$ is achieved at a detection limit $491.2 \mathrm{pW}$ in the $5 \mathrm{~L}$ device, while an improved maximum responsivity of $1.24 \times 10^{5} \mathrm{~A} \mathrm{~W}^{-1}$ with detection limit $8.86 \mathrm{pW}$ is achieved in the $10 \mathrm{~L}$ device. This detection limit of the 
10L device is comparable to the $\mathrm{PbS} /$ graphene hybrid phototransistor. ${ }^{25}$ Since graphene transistor has a weak photoresponse, the high responsivities of the devices are mainly attributed to the $\mathrm{AgBiS}_{2}$ NCs. Table 1 summarizes the device performance of this work and PbS, Si NCs IR photodetectors in the literature..$^{3,5,6,26}$ The photo-responsivities were normalized to $V_{\text {DS }}$ to eliminate the contribution of the working voltage $V_{\mathrm{DS}}$ to $R$. The performance of our $\mathrm{AgBiS}_{2} /$ graphene transistors are comparable with the PbS-based photodetectors.

\section{Conclusions}

In summary, we have successfully synthesized $\mathrm{AgBiS}_{2} \mathrm{NCs}_{\text {in }}$ an ambient environment without any observable by-products or oxide content. The NCs are highly dispersive in toluene which makes the NCs solution very suitable for solution processable device. The $\mathrm{AgBiS}_{2}$ NCs was introduced to the graphene transistor, which showed high responsivity of $10^{3}$ to $10^{5} \mathrm{~A} \mathrm{~W}^{-1}$ with infrared illumination.

\section{Conflicts of interest}

There are no conflicts to declare.

\section{Acknowledgements}

CHM acknowledges the support of the Hong Kong PhD Fellowships scheme. This work was financially supported by and PolyU grants (Project no. 4-BCAK and 1-ZVGH). The authors acknowledge Dr Cao Xie and Prof. Feng Yan for device characterization.

\section{References}

1 R. Saran and R. J. Curry, Nat. Photonics, 2016, 10, 81.

2 Y. Cao, A. Stavrinadis, T. Lasanta, D. So and G. Konstantatos, Nat. Energy, 2016, 1, 16035.

3 D. Kufer, I. Nikitskiy, T. Lasanta, G. Navickaite, F. H. Koppens and G. Konstantatos, Adv. Mater., 2015, 27, 176-180.

4 C. Chen, H. Qiao, S. Lin, C. M. Luk, Y. Liu, Z. Xu, J. Song, Y. Xue, D. Li, J. Yuan, et al., Sci. Rep., 2015, 5, 11830.
5 G. Konstantatos, M. Badioli, L. Gaudreau, J. Osmond, M. Bernechea, D. F. P. G. Arquer, F. Gatti and F. H. Koppens, Nat. Nanotechnol., 2012, 7, 363.

6 L. Gao, C. Chen, K. Zeng, C. Ge, D. Yang, H. Song and J. Tang, Light: Sci. Appl., 2016, 5, e16126.

7 G. Flora, D. Gupta and A. Tiwari, Interdiscip. Toxicol., 2012, 5, 47-58.

8 M. Bernechea, N. C. Miller, G. Xercavins, D. So, A. Stavrinadis and G. Konstantatos, Nat. Photonics, 2016, 10, 521.

9 S. N. Guin and K. Biswas, Chem. Mater., 2013, 25, 3225.

10 F. Xia, T. Mueller, Y. Lin, A. Valdes-garcia and P. Avouris, Nat. Nanotechnol., 2009, 4, 839.

11 S. F. Shi, X. Xu, D. C. Ralph and P. L. McEuen, Nano Lett., 2011, 11, 1814.

12 T. Mueller, F. Xia and P. Avouris, Nat. Photonics, 2010, 4, 297. 13 R. R. Nair, P. Blake, A. N. Grigorenko, K. S. Novoselov, T. J. Booth, T. Stauber, N. M. R. Peres and A. K. Geim, Science, 2008, 320, 1308.

14 G. Ruan, Z. Sun, Z. Peng and J. M. Tour, ACS Nano, 2011, 5, 7601.

15 F. Schwierz, Nat. Nanotechnol., 2010, 5, 487.

16 D. H. Kwak, D. H. Lim, H. S. Ra, P. Ramasamy and J. S. Lee, RSC Adv., 2016, 6, 65252.

17 G. Konstantatos, M. Badioli, L. Gaudreau, J. Osmond, M. Bernechea, F. P. G. De Arquer, F. Gatti and F. H. L. Koppens, Nat. Nanotechnol., 2012, 7, 363.

18 Z. Ni, L. Ma, S. Du, Y. Xu, M. Yuan, H. Fang, Z. Wang, M. Xu, D. Li, J. Yang, et al., ACS Nano, 2017, 11, 9854-9862.

19 M. V. Kovalenko, M. Scheele and D. V. Talapin, Science, 2009, 324, 1417.

20 R. Wang, Y. Shang, P. Kanjanaboos, W. Zhou, Z. Ning and E. H. Sargent, Energy Environ. Sci., 2016, 9, 1130.

21 J. Tang, K. W. Kemp, S. Hoogland, K. S. Jeong, H. Liu, L. Levina, M. Furukawa, X. Wang, R. Debnath, D. Cha, et al., Nat. Mater., 2011, 10, 765.

22 C. Xie, C. H. Mak, X. Tao and F. Yan, Adv. Funct. Mater., 2017, 27, 1603886.

23 D. Kufer and G. Konstantatos, ACS Photonics, 2016, 3, 2197. 24 C. Xie and F. Yan, ACS Appl. Mater. Interfaces, 2017, 9, 1569. 25 Z. Sun, Z. Liu, J. Li, G. Tai, S. P. Lau and F. Yan, Adv. Mater., 2012, 24, 5878.

26 Y. Yu, Y. Zhang, X. Song, H. Zhang, M. Cao, Y. Che, H. Dai, J. Yang, H. Zhang and J. Yao, ACS Photonics, 2017, 4, 950. 